\title{
Evaluation of liposomal carnosine in adjuvant arthritis
}

\author{
Lukáš Slovák $^{1,2}$, Silvester Poništ ${ }^{1}$, Tatiana Fedorova ${ }^{3}$, Anna Logvinenko $^{3}$, Irina Levacheva ${ }^{4}$, \\ Olga Samsonova ${ }^{4}$, Udo Bakowsky ${ }^{4}$, Ludmila Pašková ${ }^{5}$, Tomáš Čavojský ${ }^{5}$, Lia Tsiklauri ${ }^{6}$ \\ and Katarína Bauerová ${ }^{1,7}$ \\ ${ }^{1}$ Institute of Experimental Pharmacology and Toxicology of the Slovak Academy of Sciences, Dúbravská cesta 9, Bratislava, \\ Slovakia \\ ${ }^{2}$ Comenius University Bratislava, Jessenius Faculty of Medicine, Department of Pharmacology, BioMed Martin, Slovakia \\ ${ }^{3}$ Research Center of Neurology, Moscow, Volokolamskoye shosse 80, 125367 Moscow, Russian Federation \\ ${ }^{4}$ Philipps Universität Marburg, Institut für Pharmazeutische Technologie und Biopharmazie, Ketzerbach 63, D-35032 Marburg, \\ Germany \\ ${ }^{5}$ Comenius University in Bratislava, Faculty of Pharmacy, Department of Cell and Molecular Biology of Drugs, Bratislava, \\ Slovakia \\ ${ }^{6}$ Pharmaceutical Technology Department I., Kutateladze Institute of Pharmacochemistry, P. Sarajishvili st 36, Tbilisi 0159, \\ Georgia \\ ${ }^{7}$ Comenius University in Bratislava, Faculty of Pharmacy, Department of Galenic Pharmacy, Bratislava, Slovakia
}

\begin{abstract}
Liposomal carnosine could overcome the problems associated with direct application of this peptide. Anti-inflammatory and antioxidant effects of liposomal and non-liposomal carnosine in adjuvant arthritis were compared. The experiments were done on healthy animals, untreated arthritic animals, arthritic animals with oral administration of carnosine, and with subcutaneous administration of liposomal carnosine, both administered in the same daily dose of $150 \mathrm{mg} / \mathrm{kg}$ b.w. during 28 days. Carnosine reduced hind paw volume on day 28. Both forms markedly decreased interleukin-1 $\beta$, matrix metalloproteinase- 9 and monocyte chemoattractant protein-1 (MCP-1) in plasma on day 14. Only liposomal carnosine reduced significantly MCP-1. Malondialdehyde, 4-hydroxynonenal, resistance to $\mathrm{Fe}^{2+}$-induced oxidation and protein carbonyls were significantly corrected after administration of any form of carnosine. Liposomal carnosine corrected more effectively the oxidative stress in plasma than did carnosine. In brain tissue, our results showed protective ability of both forms of carnosine against oxidation of proteins and lipids. They also corrected the resistance to $\mathrm{Fe}^{2+}$-induced oxidation in arthritic animals. We found that only liposomal carnosine decreased the mRNA expression of inducible nitric oxide synthase in cartilage tissue. It can be concluded that the liposomal drug-delivery system is improving the pharmacological properties of carnosine administered in arthritis.
\end{abstract}

Key words: Liposomes - Adjuvant arthritis - Oxidative stress - Carnosine - Inflammation

\section{Introduction}

Rheumatoid arthritis (RA) is the most common autoimmune inflammatory arthritis in adults (Helmick et al. 2008). Although traditional treatment is reducing the pain

Correspondence to: Katarina Bauerova, Institute of Experimental Pharmacology and Toxicology, Slovak Academy of Sciences, Dubravska cesta 9, 84104 Bratislava, Slovakia

E-mail: katarina.bauerova@savba.sk without toxic pharmacological consequences, it is ineffective in reversing cartilage degradation and only slows it down. Moreover, its potential side effects limit the length of therapy (Cameron et al. 2009). There is thus an urgent need to find new methods and strategies for the treatment of RA. Several researchers reported that antioxidants are potential agents in RA therapeutic management (Khojah et al. 2016; Mateen et al. 2016). In the development of adjuvant arthritis (AA) not only immunological and inflammatory pathological changes are involved, but also the redox ho- 
meostasis is shifted towards increased production of reactive oxygen species (ROS) and reactive nitrogen species (RNS). Recent evidence from animal models of RA emphasized the importance of neutrophils in the initiation and progression of AA (Cross et al. 2006). We assessed ROS production in stimulated neutrophils of arthritic rats and it was found to be increased, with maximum on day 14 and 21 of AA. Neutrophils in whole blood of AA animals reacted excessively to stimulation and produced 6-9 times more ROS (Nosal et al. 2007). We also demonstrated oxidative damage to the tissues in AA: ROS levels in the joint and the spleen were significantly elevated (Drabikova et al. 2009). The control of inflammation in arthritic patients by natural as well as synthetic antioxidants could become a relevant component of antirheumatic prevention and therapy (Bauerová and Bezek 1999). Carnosine ( $\beta$-alanyl-L-histidine) is a naturally occurring antioxidant and cell protector (Boldyrev and Johnson 2002). Carnosine is available as a dietary supplement with no known side effects or identified adverse drug interactions (Min et al. 2008). One of the reasons for the limited use of carnosine as a drug is considered to be its fast metabolism by means of serum and kidney carnosinases. These enzymes quickly decrease the level of carnosine in serum, thus preventing its long-lasting action (Pegova et al. 2000). Our previous study showed a protective effect of carnosine against oxidative damage and also its systemic anti-inflammatory effects (Ponist et al. 2016). Liposomes are nanosized artificial vesicles of spherical shape that can be produced from natural or synthetic phospholipids (Schuber et al. 1998). Encapsulation of antioxidants in liposomes has been shown to improve their therapeutic potential against oxidant-induced tissue injuries, as liposomes evidently facilitate intracellular delivery and extend the retention time of incorporated agents inside the cell. Thus antioxidant liposomes hold great promise in the treatment of many diseases in which oxidative stress plays a significant role (Zacharias 2011). Entrapment of carnosine into liposomes may represent an ameliorative approach to overcome the problems related with direct application of this antioxidant peptide by improving bioavailability in brain lipid tissues and providing protection against carnosinase.

The aim of this study was to examine anti-inflammatory and antioxidant effects of liposomal carnosine in plasma and brain in AA and to compare these effects with its nonliposomal form.

\section{Materials and Methods}

\section{Animals, experimental design and treatments}

Male Lewis rats, weighing 160-180 g, were purchased from the Breeding Farm Dobra Voda (Slovakia) and housed five per cage under standard conditions with food and water ad libitum and a 12-hour-light/12-hour-dark cycle. The experimental protocol was approved by the Ethics Committee of the Institute of Experimental Pharmacology and Toxicology and by the Slovak State Veterinary and Food Administration in accordance with the European Convention for the Protection of Vertebrate Animals Used for Experimental and Other Scientific Purposes and was found in accordance with Slovak legislation. AA was induced by a single intradermal injection of $0.1 \mathrm{ml}$ suspension of heatinactivated Mycobacterium butyricum (Difco Laboratories, Detroit, MI, USA) in incomplete Freund's adjuvant at the base of the tail. The experiments included four groups of animals ( 8 animals in each group): Group 1, healthy animals as reference controls (CO); Group 2, arthritic animals without any drug administration (AA); Group 3, arthritic animals with the administration of carnosine (CARN) $150 \mathrm{mg} / \mathrm{kg}$ b.w. in a daily oral dose during 28 days; and Group 4, arthritic animals with the administration of liposomal carnosine (CARN-L) $150 \mathrm{mg} / \mathrm{kg}$ b.w. in a daily subcutaneous dose during 28 days. Body weight of the rats was regularly measured to calculate the precise application doses before each administration of the given substance. On day 14 , blood samples were taken from the retro-orbital plexus under Zoletil ${ }^{\circledast / x y l a z i n e ~ a n e s t h e s i a . ~ O n ~ d a y ~ 28, ~ t h e ~}$ animals were sacrificed under the same anesthesia and blood for plasma preparations was withdrawn along with brain and cartilage tissue from each rat. All samples were stored at $-80^{\circ} \mathrm{C}$ until biochemical analysis.

\section{Preparation of liposomes by lipid film hydration}

Liposomes were prepared by thin film hydration method. Briefly, sterol lipids and fatty acids were dissolved in an organic solvent to assure a homogeneous lipid mixture. The solution was prepared at $10-20 \mathrm{mg}$ lipid/ml of organic solvent (ethanol 100\%). Then the mixture was evaporated in a rotary evaporator. Carnosine was added to the obtained lipid film in buffer PBS (phosphate buffered saline) solution and finally the heterogeneous system was thoroughly mixed using ultrasonic equipment to obtain liposomes (the ratio drug/lipids was $0.2: 1$ ). All solutions were sterile and the preparation of liposomes was performed in sterile conditions.

\section{Clinical parameter: change of hind paw volume}

The hind paw volume (HPV, \%) increase was calculated as the percentage increase in the HPV on a given experimental day relative to the HPV at the beginning of the experiment. This parameter was recorded on days 1, 14, 21, and 28 with the use of an electronic water plethysmometer (UGO BASILE, Comerio-Varese, Italy). 
Measurement of monocyte chemoattractant protein-1 (MCP-1) in plasma

For determination of plasmatic concentration of MCP-1 an ELISA kit from eBioscience was used. The assay procedure was applied as described in the product manual. An anti-rat MCP-1 monoclonal coating antibody was adsorbed onto microwells. Rat MCP-1 was present in the sample or standard bound to antibodies adsorbed to the microwell; a biotin-conjugated monoclonal anti-rat MCP-1 antibody bound to rat MCP-1 was captured by the first antibody. Streptavidin-HRP was bound to the conjugated anti-rat MCP-1. Following incubation, unbound biotin conjugated anti-rat MCP-1 and Streptavidin-HRP were removed during a wash step and substrate solution reactive with HPR was added to the wells. A colored product was formed in proportion to the amount of soluble rat MCP-1 present in the sample. The reaction was terminated by addition of acid and absorbance was measured at $450 \mathrm{~nm}$ in comparison with reference wavelength $620 \mathrm{~nm}$ (microplate reader MRX II, Dynex, USA). The results were calculated from standard calibration curve on internal standards.

Measurement of interleukin $1 \beta(I L-1 \beta)$ and matrix metalloproteinase 9 (MMP-9) in plasma

For determination of plasmatic concentration of IL- $1 \beta$ and MMP-9, an ELISA kit from R\&D Systems Quantikine was used. The assay procedures were applied as described in the product manuals.

\section{Chemiluminescence measurement of plasma and brain homogenate antioxidant capacity}

The method used was based on the measurement of chemiluminescence $(\mathrm{ChL})$ in brain homogenates and plasma of the experimental animals oxidized by $\mathrm{Fe}^{2+}$ ions added in excess to the reaction medium (Fedorova et al. 1999). To initiate lipid peroxidation (LPO), $2.5 \mathrm{mM} \mathrm{Fe}^{2+}$ ions were added. The initial ChL burst $(h, m V)$ represents the level of pre-existent LPO products (reflects the stationary level of lipid hydroperoxides), the lag period of $\mathrm{Fe}^{2+}$-induced oxidation $(\tau, s)$ characterizes the resistance of the sample against oxidation, dependent on the intrinsic antioxidant capacity of a biological sample. ChL signal was monitored using LKB 1251Chemiluminometer (Sweden) and was expressed in $\mathrm{mV}$ (Dobrota et al. 2005).
Measurement of malondialdehyde in plasma and brain homogenates

Malondialdehyde (MDA) ELISA Kit (Cusabio cat № CSB$\mathrm{E} 08557 \mathrm{~h}$ ) for the quantitative determination of endogenic MDA concentrations in plasma and tissue homogenates was used. The assay employs an inhibition enzyme immunoassay technique. Antibody specific for MDA was pre-coated onto a microplate. Standards and samples were pipetted into the wells with horseradish-peroxidase (HRP) -conjugated MDA. A competitive inhibition reaction between MDA (standards and samples) and HRP-conjugated MDA with the pre-coated antibody specific for MDA was launched - the higher the amount of MDA in samples, the less antibody bound by HRP-conjugated MDA will bind. Following a wash to remove any unbound reagent, a substrate solution was added to the wells and color was developed. The color development was stopped and the intensity was measured.

\section{Measurement of 4-hydroxynonal and protein carbonyls in plasma and brain homogenates}

Determination of oxidative stress parameters was done by using protein carbonyl ELISA Kit (Immundiagnostik AG cat№ K7822) and 4-hydroxynonenal (HNE) ELISA Kit (Cusabio cat № CSB-E16214h). The assay procedures were applied as described in the product manuals.

\section{Total RNA isolation and quantitative RT-PCR}

Total RNA was isolated from the rat cartilage tissue. The cartilage was pulverized in liquid nitrogen followed by RNA extraction with the RNAzol RT (Sigma-Aldrich). RNA was converted into complementary DNA (cDNA) using the PrimeScript RT Reagent Kit (Takara) following the protocols of the manufacturers. Amplification and detection of cDNA of reference and target genes were performed on a 7300 Real-Time PCR System (Applied Biosystems) using HOT FIREPolEvaGreenR qPCR MixPlus (ROX) (Solis Biodyne). Relative mRNA expressions of inducible nitric oxide synthase (iNOS) were analyzed using the $\Delta \Delta$ Ct value method (Winer et al. 1999). $\beta$-actin was used as a reference gene. PCR products were evaluated by melting curve analysis to confirm the specific amplification. The sequences of the primers were designed and checked using Primer 3 and Oligo Analyzer 1.0.3 (Table 1).

Table 1. Primer sequences

\begin{tabular}{lll}
\hline Product & Sense primer $\left(5^{\prime}-3^{\prime}\right)$ & Antisense primer $\left(5^{\prime}-3^{\prime}\right)$ \\
\hline iNOS & AAAACCCCAGGTGCTATTCCC & GTGGTGAAGGGTGTCGTGAA \\
$\beta$-actin & CCGCGAGTACAACCTTCTTG & GCAGCGATATCGTCATCCA \\
\hline
\end{tabular}




\section{Statistical analyses}

Mean and S.E.M. values were calculated for each parameter in each group. Statistically significant differences among treated groups, untreated group and control group were tested using parametric Analysis of variance (ANOVA). After post hoc (Tukey-Kramer) testing, significance designations were specified as follows: extremely significant (** $p<0.001)$, highly significant $\left({ }^{* *} p<0.01\right)$, significant $\left({ }^{*} p<0.05\right)$, and not significant $(p>0.05)$.

\section{Results}

\section{Clinical parameter}

HPV of arthritic animals increased during the experiment in comparison to the control group on all days monitored. The highest increase was observed at the end of the experiment - day 28. Carnosine and liposomal carnosine reduced this parameter, but this effect was not statistically significant, except on day 28 when carnosine significantly reduced HPV (Fig. 1).

\section{Inflammatory parameters in plasma evaluated on day 14}

The level of IL-1 $\beta$ in untreated arthritic animals was increased approximately 12 times on day 14 when compared to healthy controls. Carnosine and liposomal carnosine

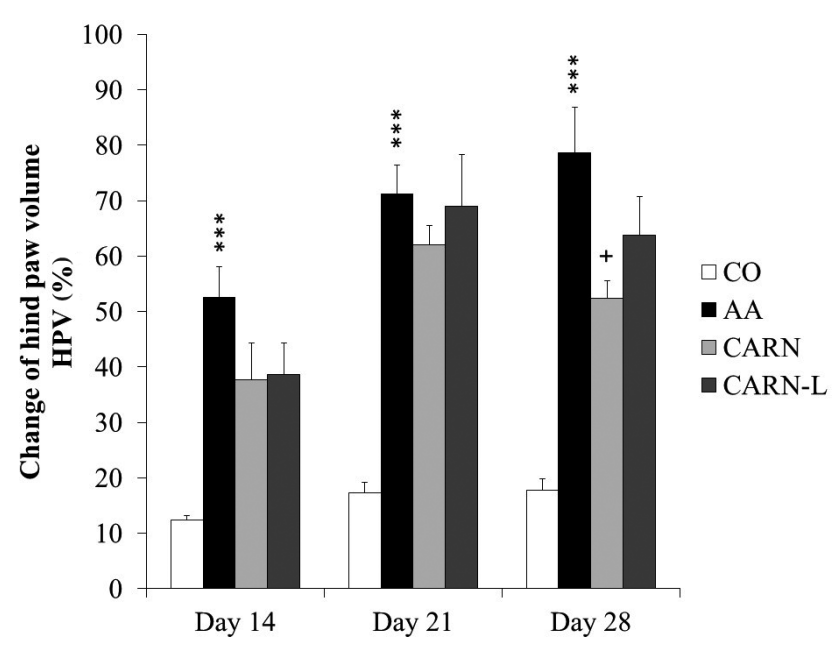

Figure 1. Change of hind paw volume (HPV) in adjuvant arthritis and the effect of carnosine and liposomal carnosine. Data are expressed as mean \pm SEM; $n=8 .^{* * *} p<0.001 v s$. healthy control, $+p<0.05$ vs. AA group. CO, control group; AA, adjuvant arthritis group; CARN, adjuvant arthritis group with administered carnosine; CARN-L, adjuvant arthritis group with administered liposomal carnosine. significantly decreased IL- $1 \beta$ concentration in plasma to the basal level analyzed in healthy animals (Table 2). Further arthritis significantly increased MMP-9 and MCP-1 in plasma on day 14 (Table 2 and Fig. 2). Carnosine and liposomal carnosine were effective in reducing significantly the MMP-9 levels (Table 2). Concerning MCP-1 in plasma, only liposomal carnosine (Fig. 2) reduced the levels of this parameter significantly. Thus liposomal carnosine was generally more effective in improving the inflammation analyzed in plasma than carnosine.

\section{Antioxidant capacity of carnosine and liposomal carnosine in plasma and brain tissue homogenate}

Plasma: Neither AA nor carnosine administered changed the content of lipid hydroperoxide (data not shown). However, AA significantly reduced the resistance to $\mathrm{Fe}^{2+}$ induced oxidation and both liposomal and non-liposomal carnosine were able to effectively increase it. In comparison to carnosine, liposomal carnosine was much more effective (Fig. 3). Oxidation of plasmatic proteins was assayed as protein carbonyls, which were increased in untreated arthritic animals. Carnosine and its liposomal form administered decreased effectively the protein carbonyls to control level. The arthritic process increased lipid peroxidation in plasma detected as levels of MDA and HNE. Carnosine and liposomal carnosine reduced the levels of MDA and both forms of carnosine studied were also able to decrease HNE to control level (Table 3).

Brain: The content of lipid hydroperoxides was increased in animals with AA without significance. Carnosine and

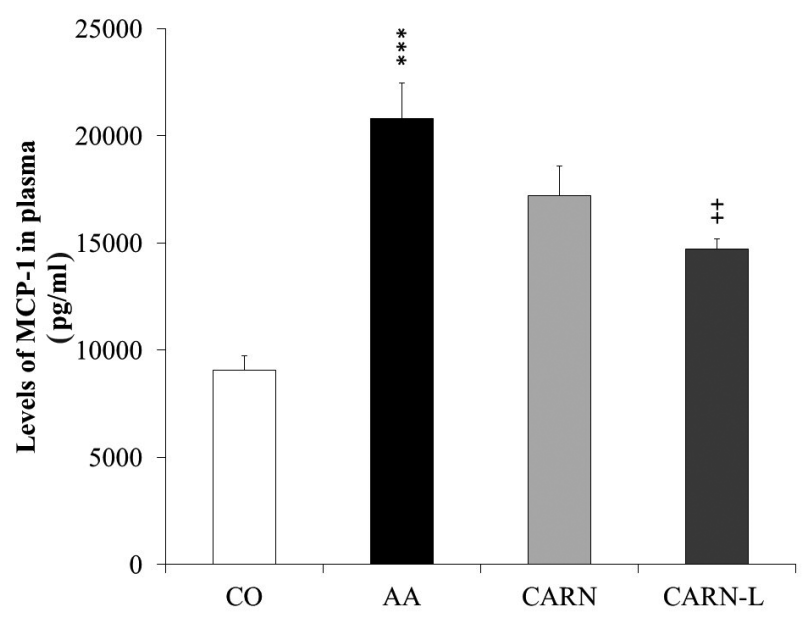

Figure 2. Inflammatory marker monocyte chemoattractant protein-1 MCP-1 measured in plasma on day 14. Data are expressed as mean \pm SEM; $n=8 .{ }^{* * *} p<0.001 v s$. healthy control, ${ }^{++} p<0.05$ $v s$. AA group. For abbreviations, see Fig. 1. 
Table 2. Parameters of inflammation measured in plasma on day 14. Level of interleukin $1 \beta$ (IL-1 $\beta$ ) and matrix metalloproteinase 9 (MMP-9)

\begin{tabular}{lrccc}
\hline \multirow{2}{*}{ Inflammatory parameters } & \multicolumn{4}{c}{ Group } \\
\cline { 2 - 5 } & \multicolumn{5}{c}{ CO } & AA & CARN & CARN-L \\
\hline IL-1 $\beta(\mathrm{pg} / \mathrm{ml})$ & $7.46 \pm 0.75$ & $94.49 \pm 26.32^{* *}$ & $17.24 \pm 3.05^{++}$ & $17.18 \pm 3.90^{++}$ \\
MMP-9 $(\mathrm{ng} / \mathrm{ml})$ & $37.88 \pm 3.55$ & $133.57 \pm 9.31^{\star * *}$ & $60.59 \pm 6.18^{+++}$ & $64.55 \pm 9.17^{+++}$ \\
\hline
\end{tabular}

$\mathrm{CO}$, control group; AA, adjuvant arthritis group; CARN, adjuvant arthritis group administered with carnosine; CARN-L, adjuvant arthritis group administered with liposomal carnosine. Data are expressed as mean $\pm \mathrm{SEM} ; n=8 .{ }^{* *} p<0.01 v s$. CO, ${ }^{* *} p<0.001$ vs. CO, ${ }^{++} p<0.01 v s . \mathrm{AA},{ }^{+++} p<0.001 v s . \mathrm{AA}$.

liposomal carnosine administered decreased the signal for damaged lipids (data not shown). Arthritis in experimental animals significantly reduced the resistance to $\mathrm{Fe}^{2+}$-induced oxidation and both forms of carnosine administered were able to increase it effectively, with liposomal carnosine being even more effective (Fig. 3). Protein carbonyls were increased in the brain of untreated arthritic animals, though not significantly. Carnosine and liposomal carnosine decreased brain protein carbonyls. Lipid peroxidation in the brain measured as content of MDA was increased 2-times in arthritic animals in comparison to healthy control. Both forms of carnosine administered reduced the content of MDA to control level. Moreover, HNE content was increased due to arthritis. Carnosine and liposomal carnosine were able to decrease this parameter significantly (Table 4).

Generally, the antioxidant effect of liposomal carnosine in plasma was stronger than that of carnosine. This trend was not observed in the brain.

\section{Effect of carnosine and liposomal carnosine on mRNA expression of iNOS in rat cartilage tissue}

In our study, the expression of iNOS mRNA was significantly increased in untreated arthritic animals (Fig. 4). The administration of carnosine did not lead to changes in mRNA expression of iNOS. Liposomal carnosine reduced this parameter without statistical significance.

\section{Discussion}

We intend to find new substances of natural origin with anti-inflammatory and antioxidant effect in AA. In an experimental study (Ponist et al. 2016) we investigated the effect of carnosine in vitro and in vivo, using two different animal models. The results indicated its potential systemic anti-inflammatory effects. It still remains unclear if the ability of carnosine to restore redox balance is the only mechanism

Table 3. Parameters of oxidative stress measured in plasma on day 28. Level of protein carbonyls, malondialdehyde (MDA) and 4-hydroxynonenal (HNE)

\begin{tabular}{lcccc}
\hline & \multicolumn{4}{c}{ Group } \\
\cline { 2 - 5 } & CO & AA & CARN & CARN-L \\
\hline Carbonyls $(\mu \mathrm{g} / \mathrm{ml})$ & $391.2 \pm 14.34$ & $457.72 \pm 11.09^{* * *}$ & $393.36 \pm 2.21^{+++}$ & $392.62 \pm 3.6^{+++}$ \\
MDA $(\mu \mathrm{g} / \mathrm{ml})$ & $2.4 \pm 0.39$ & $5.79 \pm 0.44^{* * *}$ & $2.9 \pm 0.25^{++}$ & $2.11 \pm 0.32^{+++}$ \\
HNE $(\mathrm{ng} / \mathrm{ml})$ & $1.54 \pm 0.16$ & $2.5 \pm 0.19^{* * *}$ & $1.5 \pm 0.07^{+++}$ & $1.55 \pm 0.09^{+++}$ \\
\hline
\end{tabular}

Data are expressed as mean $\pm \mathrm{SEM} ; \mathrm{n}=8 .{ }^{* *} p<0.001$ vs. $\mathrm{CO},{ }^{++} p<0.01$ vs. AA, ${ }^{+++} p<0.001$ vs. AA. For abbreviations, see Tab. 2.

Table 4. Parameters of oxidative stress measured in brain on day 28. Content of protein carbonyls, malondialdehyde (MDA) and 4-hydroxynonenal (HNE)

\begin{tabular}{lcccc}
\hline & \multicolumn{4}{c}{ Group } \\
\cline { 2 - 4 } & CO & AA & CARN & CARN-L \\
\hline Carbonyls $(\mu \mathrm{g} / \mathrm{g}$ tissue) & $548.07 \pm 15.0$ & $606.12 \pm 74.2$ & $475.4 \pm 10.6^{+}$ & $470.2 \pm 8.0^{+}$ \\
MDA $(\mu \mathrm{g} / \mathrm{g}$ tissue) & $5.38 \pm 0.73$ & $10.12 \pm 1.01^{* * *}$ & $3.45 \pm 0.23^{+++}$ & $5.52 \pm 0.8^{+++}$ \\
HNE $(\mathrm{ng} / \mathrm{g}$ tissue) & $3.26 \pm 0.17$ & $4.78 \pm 0.5^{* *}$ & $3.22 \pm 0.15^{++}$ & $3.54 \pm 0.23^{+}$ \\
\hline Data are expressed as mean \pm SEM; $\mathrm{n}=8^{* *} p<0.01 v s . \mathrm{CO},{ }^{* * *} p<0.001 v s . \mathrm{CO}^{+}{ }^{+} p<0.05 v s$. AA, ${ }^{++} p<0.01 v s$. AA, ${ }^{+++} p<0.001 v s$. \\
AA. For abbreviations, see Tab. 2.
\end{tabular}




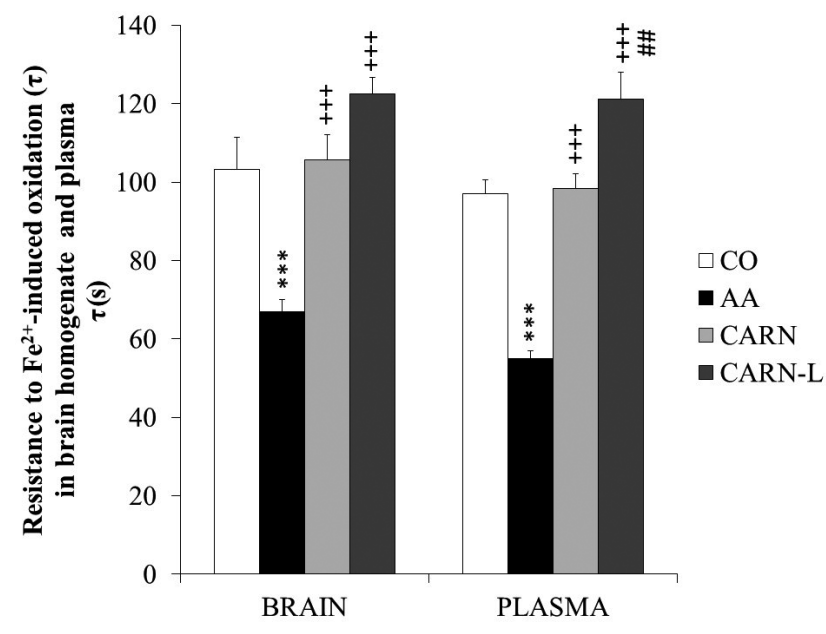

Figure 3. Resistance to $\mathrm{Fe}^{2+}$-induced oxidation $(\tau)$ in brain homogenate and plasma on day 28 . Data are expressed as mean \pm SEM; $n=8 .{ }^{* * *} p<0.001 v s$. healthy control, ${ }^{+++} p<0.001 v s$. AA group, ${ }^{\# \#} p<0.01$ vs. CARN group. For abbreviations, see Fig. 1.

responsible for its anti-inflammatory effects in AA. One goal of this research was to assess whether drug-delivery systems, such as liposomes, could improve the therapeutic potency and bioavailability of carnosine.

Several researchers have focused on the application of liposomes as drug carriers in the treatment of RA (Kapoor et al. 2014). In this study, we monitored AA progression by using the change of HPV. Liposomal carnosine reduced HPV change on both experimental days similarly as did carnosine. Moreover, carnosine reduced hind paw edema significantly on day 28. Topical application of mannitol exerted a potent and fast anti-edema effect in a rat model of joint inflammation, suggesting a possible utilization in patients affected by RA (Cavone et al. 2012). Intravenous therapy with liposomal dexamethasone phosphate (DxM-P) suppressed joint swelling in a significant, dose-dependent, and long-lasting manner and showed superior therapeutic efficacy compared to matched doses of free DxM-P (Anderson et al. 2010). Thus liposomal encapsulation of current anti-rheumatic drugs, and of new potential substances for RA treatment, concerning also other drug-delivery systems, may clearly potentiate the clinical efficacy of the compounds evaluated.

Monitoring of cytokines, chemokines and other proinflammatory mediators which play essential roles in the progression of inflammatory diseases is very important for evaluation of the effectivity of the treatment. For determination of inflammation in our study, we assessed plasmatic levels of cytokine IL-1 $\beta$, chemokine MCP-1 and protease MMP-9. IL-1 is the first molecule occurring at high levels in the synovial tissue in this T-cell-dependent arthritis. The early phases of the disease seem to be characterized by a systemic increase in IL-1 $\beta$ (Ferraccioli et al. 2010). Monocytes, macrophages and dendritic cells, as well as neutrophils, are among the immune cells capable of producing large amounts of IL-1 $\beta$ (Wilson et al. 1994; Thornberry et al. 1992). Arthritic animals demonstrated a significant increase in neutrophil numbers on day 14 in AA (Perecko et al. 2013). It seems, that neutrophils are important producers of IL- $1 \beta$ in AA. We found in literature, that carnosine decreased more than by $50 \%$ the production of ROS measured by luminol-dependent chemiluminescence of PMA-stimulated human neutrophils (Sharonov et al. 1990). Therefore, we assume, carnosine and liposomal carnosine inhibited the activity of neutrophils and their production IL- $1 \beta$. From our results, we can conclude, liposomal form of carnosine has no effect on the ability of carnosine to reduce the level of IL-1 $\beta$. Carnosine and liposomal carnosine decreased the IL- $1 \beta$ to basal level. It is possible that both carnosine forms are affecting the function of macrophages, which produce IL- $1 \beta$. In one of our previous studies, both high- and low-molecular-weight non-animal chondroitin sulphate were effective in reducing plasmatic levels of proinflammatory cytokines in AA, including IL-1 $\beta$ (Bauerova et al. 2014). Carnosine and its liposomal form might be good candidates for decrease of IL-1 levels in patients with RA.

MCP-1 may play an important role in the recruitment of peripheral blood leukocytes into the RA joints. Data from a study performed in RA patients provide evidence that the chemokine signalling pathway is involved in MCP-1 expression in the patients' tissues, which may contribute to chronic inflammation associated with RA. Targeting this signalling pathway may provide a novel therapeutic direction in RA (Zhang et al. 2015). Liposomal carnosine significantly decreased the plasmatic level of MCP-1 on

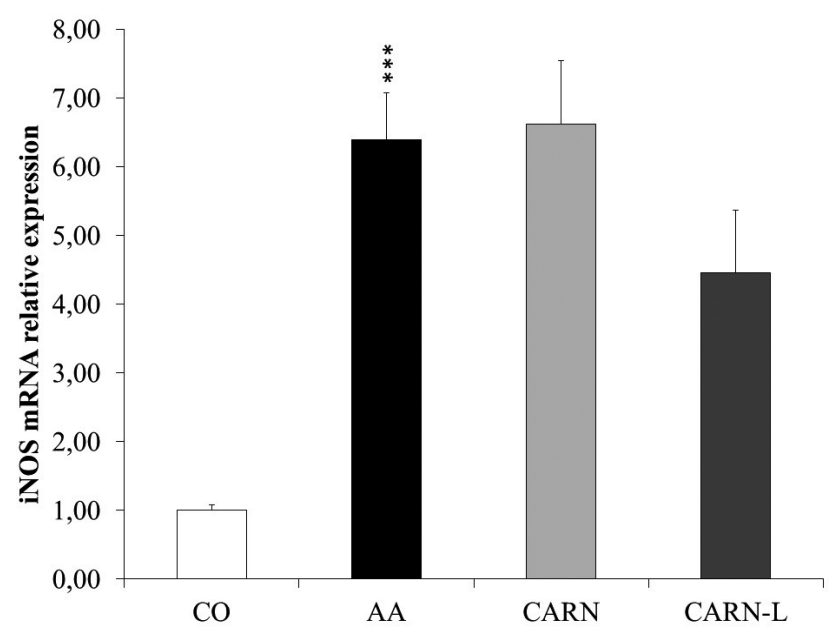

Figure 4. Relative changes of inducible nitric oxide synthase iNOS mRNA expression normalized to $\beta$-actin mRNA in arthritic rat cartilage tissue. Data are expressed as mean \pm SEM; $n=8 .{ }^{* * \star} p<$ $0.001 v s$. healthy control. For abbreviations, see Fig. 1. 
day 14 , while carnosine failed to affect it. It seems that incorporation of carnosine into liposomes increases its ability to reduce MCP-1 levels in plasma during AA development. Liposomal carnosine may play some role in the pathway of MCP-1 signalling.

Matrix metalloproteinases (MMPs) produced by macrophages and synovial fibroblasts were shown to be involved in the destruction of articular tissues in RA (Cunnane et al. 2014). Tumor necrosis factor- $\alpha$ (TNF- $\alpha$ ), interleukin- $1 \beta$ (IL$1 \beta$ ) and IL- 6 can regulate MMPs gene expression (Malemud and Schulte 2008). Since production of MMPs is controlled by TNF- $\alpha$, the use anti-TNF agents has been suggested in the treatment of RA (Klimiuk et al. 2004). In our study, both forms of carnosine were significantly effective in reducing MMP-9 in plasma. This ability of liposomal and nonliposomal carnosine might be also involved in the decrease of IL-1 $\beta$ plasmatic levels to basal values. Unfortunately, the results from the hind paw volume of animals for carnosine and liposomal carnosine do not correspond well with the intensive reduction of IL- $1 \beta$ plasmatic levels on day 14 . This might be explained by the fact that not only IL- $1 \beta$ but also TNF- $\alpha$ and IL- 6 regulate MMP-9 gene expression.

In animal models of RA, increased oxidative stress has been well documented by other authors (Wruck et al. 2011; Ahmed et al. 2015). In our study, oxidative stress in AA was analyzed by parameters in brain homogenates and in blood plasma. Lipid peroxidation is one of the major consequences of oxidative stress and leads to the production of conjugated diene hydroperoxides and unstable substances, which disintegrate into various bioactive aldehydes such as MDA and HNE (Phaniendra et al. 2015). Another important consequence of oxidative damage in RA is the deleterious effect on proteins, as free radicals can modify both their structure and functions (Dalle-Donne et al. 2006). Carnosine and liposomal carnosine significantly corrected all parameters of oxidation stress measured in plasma, except the content of lipid hydroperoxides. Liposomal carnosine was better in corrections of oxidative stress in plasma than carnosine, only in the resistance to $\mathrm{Fe}^{2+}$-induced oxidation. There was no difference in the ability of carnosine and liposomal carnosine to protect the proteins and lipids in plasma against oxidation.

$\mathrm{RA}$ is not a disease typically involving the CNS, but brain dysfunctions occur in 20 to $30 \%$ of rheumatic patients (Appenzeller et al. 2004). There are only few studies describing oxidative stress and brain damage during experimental arthritis such as AA (Skurlova et al. 2010; Skurlova et al. 2011; de Almeida Gonçalves et al. 2015). Carnosine is accumulated in excitable tissues (brain, heart and skeletal muscles) of vertebrates in large amounts (Abe 1995). In in vitro experiments carnosine protected brain neurons against oxidative injury (Boldyrev et al. 1999). Liposomes are widely used as carriers or delivery vehicles for therapeutic agents/ drugs to transport them to specific sites inside the human body (e.g. brain). Liposomes could be the carrier system for the drugs, they do not cross the blood brain barrier (Spuch and Navarro 2011). We therefore evaluated the effect of liposomal and non-liposomal carnosine on oxidative stress in brain homogenates. Our results with carnosine and liposomal carnosine showed protective ability of both forms of carnosine against protein and lipid oxidation. They corrected the resistance to $\mathrm{Fe}^{2+}$-induced oxidation in arthritic animals. Liposomal carnosine was more effective than carnosine but the difference was without significance.

We also evaluated mRNA expression of iNOS in the cartilage tissue of rats with AA. The inducible isoform of NOS is found in the synovial tissue and cartilage and its expression is regulated by catabolic cytokines, such as IL- $1 \beta$ and TNF- $\alpha$ (Amin et al. 1999). A preclinical study indicated that NO generation by iNOS induced in chondrocytes was a key event in the induction of AA (Yonekura et al. 2003). The results obtained in our experiment showed that only the liposomal form of carnosine decreased mRNA expression of iNOS in AA, and that not significantly. Liposomes can be assumed to improve bioavailability of carnosine in the rat cartilage tissue.

To the best of our knowledge, this is the first report in literature evaluating liposomal carnosine in AA. In an animal model of RA, the obtained results demonstrated that subcutaneously administered liposomal carnosine ameliorated all parameters of inflammation and oxidative stress measured. Moreover, in comparison with its non-liposomal form, liposomal carnosine was more effective in several parameters, especially MCP-1, the resistance to $\mathrm{Fe}^{2+}$-induced oxidation in plasma and brain homogenates, and the mRNA expression of iNOS in rat cartilage tissue. Liposomal carnosine showed a beneficial effect in AA, pointing to its potential use in the treatment of RA in humans.

Acknowledgements. This work was supported by the grant from VEGA 2/0044/15, APVV-15-0308 and bilateral SAS-RAMS project 2013-2015 coordinated by Dr. Bauerova (Slovakia) and Dr. Fedorova (Russia). Special thanks are due to Ing. Danica Mihalova and Jana Urgosova for their technical assistance.

\section{References}

Abe H. (1995): Histidine-related dipeptides: distribution, metabolism and physiological function. In: Biochemistry and Molecular Biology of Fishes. (Eds. Hochachka P. W., Mommsen T. P.), pp. $310-333$ https://doi.org/10.1016/S1873-0140(06)80017-2

Ahmed Y. M., Messiha B. A., Abo-Saif A. A. (2015): Protective effects of simvastatin and hesperidin against complete Freund's adjuvant-induced rheumatoid arthritis in rats. Pharmacology 96, 217-225 https://doi.org/10.1159/000439538 
Amin A. R., Attur M., Abramson S. B. (1999): Nitric oxide synthase and cyclooxygenases: distribution, regulation, and intervention in arthritis. Curr. Opin. Rheumatol. 11, 202-209 https://doi.org/10.1097/00002281-199905000-00009

Anderson R., Franch A., Castell M., Perez-Cano F. J., Bräuer R., Pohlers D., Gajda M., Siskos A. P., Katsila T., Tamvakopoulos C., Rauchhaus U., Panzner S., Kinne R. W. (2010): Liposomal encapsulation enhances and prolongs the anti-inflammatory effects of water-soluble dexamethasone phosphate in experimental adjuvant arthritis. Arthritis Res. 12, R147 https://doi.org/10.1186/ar3089

Appenzeller S., Bertolo M. B., Costallat L. T. (2004): Cognitive impairment in rheumatoid arthritis. Methods Find. Exp. Clin. Pharmacol. 5, 339-343 https://doi.org/10.1358/mf.2004.26.5.831324

Bauerová K., Bezek A. (1999): Role of reactive oxygen and nitrogen species in etiopathogenesis of rheumatoid arthritis. Gen. Physiol. Biophys. 18, 15-20

Bauerova K., Ponist S., Kuncirova V., Drafi F., Mihalova D., Paulovicova E., Volpi N. (2014): Effect of nonanimal high- and low-molecular-mass chondroitin sulfates produced by a biotechnological process in an animal model of polyarthritis. Pharmacology 94, 109-114 https://doi.org/10.1159/000366285

Boldyrev A. A., Song R., Lawrence D., Carpenter D. O. (1999): Carnosine protects against excitotoxic cell death independently of effects on reactive oxygen species. Neuroscience 94, 571-577 https://doi.org/10.1016/S0306-4522(99)00273-0

Boldyrev A., Johnson P. (2002): Carnosine and related compounds: antioxidant dipeptides. In: Oxidative Stress at Molecular, Cellular and Organ Levels. (Eds. Boldyrev A., Johnson P.), pp. 101-113, Research Signpost, Trivandrum, India

Cameron M., Gagnier J. J., Little C. V., Parsons T. J., Bluemle A., Chrubasik S. (2009): Evidence of effectiveness of herbal medicinal products in the treatment of arthritis. Part 1: Osteoarthritis. Phytother. Res. 23, 1497-1515 https://doi.org/10.1002/ptr.3007

Cavone L., Calosi L., Cinci L., Moroni F., Chiarugi A. (2012): Topical mannitol reduces inflammatory edema in a rat model of arthritis. Pharmacology 89, 18-21 https://doi.org/10.1159/000335094

Cross A., Barnes T., Bucknall R. C., Edwards S. W., Moots R. J. (2006): Neutrophil apoptosis in rheumatoid arthritis is regulated by local oxygen tensions within joints. J. Leukoc. Biol. 80, 521-528 https://doi.org/10.1189/jlb.0306178

Cunnane G., FitzGerald O., Beeton C., Cawston T. E., Bresnihan B. (2014): Early joint erosions and serum levels of matrix metalloproteinase $\mathbf{1}$, matrix metalloproteinase $\mathbf{3}$, and tissue inhibitor of metalloproteinases 1 in rheumatoid arthritis. Arthritis Rheum. 4, 2263-2274

Dalle-Donne I., Rossi R., Colombo R., Giustarini D., Milzani A. (2006): Biomarkers of oxidative damage in human disease. Clin. Chemistry 52, 601-623 https://doi.org/10.1373/clinchem.2005.061408

de Almeida Gonçalves G., de Sá-Nakanishi A. B., Wendt M. M., Comar J. F., Bersani Amado C. A., Bracht A., Peralta R. M. (2015): Green tea extract improves the oxidative state of the liver and brain in rats with adjuvant-induced arthritis. Food Funct. 6, 2701-2711 https://doi.org/10.1039/C5FO00548E

Dobrota D., Fedorova T., Stvolinsky S., Babushikova E., Likavcanova K., Drgova A., Strapkova A., Boldyrev A. (2005): Carnosine protects the brain of rats and mongolian gerbils against ischemic injury: after-stroke-effect. Neurochem. Res. 30, 1283-1288 https://doi.org/10.1007/s11064-005-8799-7

Drabikova K., Perecko T., Nosal R., Bauerova K., Ponist S., Mihalova D., Kogan G., Jancinova V. (2009): Glucomannan reduces neutrophil free radical production in vitro and in rats with adjuvant arthritis. Pharmacol. Res. 59, 399-403 https://doi.org/10.1016/j.phrs.2009.02.003

Fedorova T. N., Boldyrev A. A., Gannushkina I. V. (1999): Lipid peroxidation in experimental ischemia of the brain. Biochemistry $64,75-79$

Ferraccioli G., Bracci-Laudiero L., Alivernini S., Gremese E., Tolusso B., de Benedetti F. (2010): Interleukin-1 and interleukin-6 in arthritis animal models: roles in the early phase of transition from acute to chronic inflammation and relevance for human rheumatoid arthritis. Mol. Med. 16, 552-557 https://doi.org/10.2119/molmed.2010.00067

Helmick C. G., Felson D. T., Lawrence R. C., Gabriel S., Hirsch R., Kwoh C. K., Liang M. H., Kremers H. M., Mayes M. D., Merkel P. A. et al. (2008): Estimates of the prevalence of arthritis and other rheumatic conditions in the United States. Part I. Arthritis Rheum. 58, 15-25 https://doi.org/10.1002/art.23177

Kapoor B., Singh S. K., Gulati M., Gupta R., Vaidya Y. (2014): Application of liposomes in treatment of rheumatoid arthritis: quo vadis. ScientificWorldJournal 2014, 978351 https://doi.org/10.1155/2014/978351

Khojah H. M., Ahmed S., Abdel-Rahman M. S., Hamza A. B. (2016): Reactive oxygen and nitrogen species in patients with rheumatoid arthritis as potential biomarkers for disease activity and the role of antioxidants. Free Radic. Biol. Med. 97, 285-291 https://doi.org/10.1016/j.freeradbiomed.2016.06.020

Klimiuk P. A., Sierakowski S., Domyslawska I., Chwiecko J. (2004): Effect of repeated infliximab therapy on serum matrix metalloproteinases and tissue inhibitors of metalloproteinases in patients with rheumatoid arthritis. J. Rheumatol. 31, 238-242

Malemud C. J., Schulte M. E. (2008): Is there a final common pathway for arthritis? Future Rheumatol. 3, 253-268 https://doi.org/10.2217/17460816.3.3.253

Mateen S., Moin S., Zafar A., Khan A. Q. (2016): Redox signaling in rheumatoid arthritis and the preventive role of polyphenols. Clin. Chim. Acta 463, 4-10 https://doi.org/10.1016/j.cca.2016.10.007

Min J., Senut M. C., Rajanikant K., Greenberg E., Bandagi R., Zemke D., Mousa A., Kassab M., Farooq M. U., Gupta R., Majid A. (2008): Differential neuroprotective effects of carnosine, anserine, and $\mathrm{N}$-acetyl carnosine against permanent focal ischemia. J. Neurosci. Res. 86, 2984-2991 https://doi.org/10.1002/jnr.21744

Nosal R., Jancinova V., Petríkova M., Ponist S., Bauerova K. (2007): Suppression of oxidative burst of neutrophils with methotrexate in rat adjuvant arthritis. Chem. Letters 101, 243-244 
Pegova A., Abe H., Boldyrev A. (2000): Hydrolysis of carnosine and related compounds. Comp. Biochem. Physiol. B. Biochem. Mol. Biol. 127, 443-446 https://doi.org/10.1016/S0305-0491(00)00279-0

Perecko T., Drabikova K., Lojek A., Ciz M., Ponist S., Bauerova K., Nosal R., Harmatha J., Jancinova V. (2013): The effects of pterostilbene on neutrophil activity in experimental model of arthritis. Biomed. Res. Int. 2013, 106041 https://doi.org/10.1155/2013/106041

Phaniendra A., Jestadi D. B., Periyasamy L. (2015): „Free radicals: properties, sources, targets, and their implication in various diseases“. Indian J. Clin. Biochem. 30, 11-26 https://doi.org/10.1007/s12291-014-0446-0

Ponist S., Drafi F., Kuncirova V., Mihalova D., Rackova L., Danisovic L., Ondrejickova O., Tumova I., Truova O., Fedorova T., Bauerova K. (2016): Effect of carnosine in experimental arthritis and on primary culture chondrocytes. Oxid. Med. Cell. Longev. 2016, 8470589 https://doi.org/10.1155/2016/8470589

Schuber F., Kichler A., Boeckler C., Frisch B. (1998): Liposomes: from membrane models to gene therapy. Pure Appl. Chem. 70, 89-96 https://doi.org/10.1351/pac199870010089

Sharonov B. P., Govorova N. J., Lyzlova S. N. (1990): Carnosine as a potential scavenger of oxidants generated by stimulated neutrophils. Biochem. Int. 21, 61-68

Skurlova M., Stofkova A., Jurcovicova J. (2011): Anxiety-like behavior in the elevated-plus maze tests and enhanced IL-1 $\beta$, IL-6, NADPH oxidase- 1 , and iNOS mRNAs in the hippocampus during early stage of adjuvant arthritis in rats. Neurosci. Lett. 487, 250-254 https://doi.org/10.1016/j.neulet.2010.10.032

Skurlova M., Stofkova A., Kiss A., Belacek J., Pecha O., Deykun K., Jurcovicova J. (2010): Transient anorexia, hyper-nociception and cognitive impairment in early adjuvant arthritis in rats. Endocr. Regul. 44, 165-173 https://doi.org/10.4149/endo_2010_04_165

Spuch C., Navarro C. (2011): Liposomes for targeted delivery of active agents against neurodegenerative diseases (Alzheimer's disease and Parkinson's disease). J. Drug Deliv. 2011, 469679 https://doi.org/10.1155/2011/469679
Thornberry N.A., Bull H. G., Calaycay J. R., Chapman K. T., Howard A. D., Kostura M. J., Miller D. K., Molineaux S. M., Weidner J. R., Aunins J. et al. (1992): A novel heterodimeric cysteine protease is required for interleukin- $1 \beta$ processing in monocytes. Nature 356, 768-774 https://doi.org/10.1038/356768a0

Wilson K. P., Black J. A., Thomson J. A., Kim E. E., Griffith J. P., Navia M. A., Murcko M. A., Chambers S. P., Aldape R. A., Raybuck S. A. et al. (1994): Structure and mechanism of interleukin- $1 \beta$ converting enzyme. Nature 370, 270-275 https://doi.org/10.1038/370270a0

Winer J., Jung C. K. S., Shackel I., Williams P. M. (1999): Development and validation of real-time quantitative reverse transcriptase-polymerase chain reaction for monitoring gene expression in cardiac myocytes in vitro. Anal. Biochem. 270, 41-49 https://doi.org/10.1006/abio.1999.4085

Wruck C. J., Fragoulis A., Gurzynski A., Brandenburg L. O., Kan Y. W., Chan K., Hassenpflug J., Freitag-Wolf S., Varoga D., Lipross S., Pufe T. (2011): Role of oxidative stress in rheumatoid arthritis: insights from the Nrf2-knockout mice. Ann. Rheum. Dis. 70, 844-850 https://doi.org/10.1136/ard.2010.132720

Yonekura Y., Koshiishi I., Yamada K., Mori A., Uchida S., Nakamura T., Utsumi H. (2003): Association between the expression of inducible nitric oxide synthase by chondrocytes and its nitric oxide-generating activity in adjuvant arthritis in rats. Nitric Oxide 8, 164-169 https://doi.org/10.1016/S1089-8603(03)00025-9

Zacharias E. (2011): Suntres liposomal antioxidants for protection against oxidant-induced damage. J. Toxicol., 152474

Zhang L., Yu M., Deng J., Lv X., Liu J., Xiao Y., Yang W., Zhang Y., Li C. (2015): Chemokine signaling pathway involved in CCL2 expression in patients with rheumatoid arthritis. Yonsei Med. J. 56, 1134-1142 https://doi.org/10.3349/ymj.2015.56.4.1134

Received: March 8, 2017

Final version accepted: April 3, 2017

First published online: August 24, 2017 